\title{
Fundamental solution of bond pricing in the Ho-Lee stochastic interest rate model under the invariant criteria
}

\author{
Burhaneddin Izgi ${ }^{1}$ and Ahmet Bakkaloglu ${ }^{2}$ \\ ${ }^{1}$ Department of Mathematics, Istanbul Technical University, Turkey \\ ${ }^{2}$ Department of Mathematics, Mimar Sinan Fine Arts University, Turkey
}

Received: 13 February 2017, Accepted: 27 February 2017

Published online: 19 March 2017.

\begin{abstract}
We study the fundamental solution of bond-pricing in the Ho-Lee stochastic interest rate model under the invariant criteria. We obtain transformations between Ho-Lee model with the corresponding linear $(1+1)$ partial differential equation and the first Lie canonical form which is identical to the classical heat equation. These transformations help us to generate the fundamental solution for the Ho-Lee model with respect to the fundamental solution of the classical heat equation sense. Moreover, as a financial application of the Ho-Lee model, we choose the drift term from power functions and perform simulations via Milstein method. Furthermore, we obtain important results for the parameter calibration of the corresponding drift term by using the simulation results.
\end{abstract}

Keywords: Ho-Lee stochastic interest rate model, heat equation, canonical Lie forms, Lie symmetry analysis, invariant criteria, simulations.

\section{Introduction}

Interest rate have an important role not only in financial theory but also for the risky players in the market. Our aim in this paper is to present fundamental solution of bond pricing in the Ho-Lee stochastic interest rate model and show the transformation between Ho-Lee stochastic interest rate model and the classical heat equation via the invariant criteria for the Lie canonical forms. Our approach in this paper is in the line of the Mahomed's paper [8] which is done onto the completeness of the invariant characterization of scalar (1+1) parabolic equations. On the other hand, in the applications of the Ho-Lee model the drift term's is the one of the most important term. For this purpose, we perform simulations for the power function in the drift term of the Ho-Lee model and present the calibration of it with the suitable parameter choice.

Recently, Lie symmetry applications to stochastic differential equations (SDEs), which use in mathematical finance, have attracted more attention in the literature. This approach helps to explain sophisticated SDEs in the view of the analytic or numerical solutions for partial differential equations (PDEs) whose solutions are already known. The seminal work of Lie symmetry analysis to SDE has been done for Merton-Black Scholes model [9] by Gazizov and Ibrahimov in 1998 [3]. In 2000, Goard [4] provided an alternative solutions for bond pricing PDEs using Lie's classical method. The bond pricing problems are worked by Pooe, Mahomed and Soh [12] in 2004.

On the other hand, the group invariant solutions and an optimal system of the bond prices for the Cox-Ingersoll-Ross (CIR) model is investigated by Sinkala, Leach and Hara in [13], 2008. Moreover, Dimas et al. consider Black-Scholes, Longstaf, Vasicek, CIR and Heath equations to discover connections with the heat equations by the means of Lie symmetry in 2009 [2]. Mahomed et al. show utilization of the invariant criteria for linear one time variable and one space variable parabolic PDEs in finance and present new approach by the Hamiltonian viewpoint [7]. 
Furthermore, we present relationship between the Ho-Lee model and classical heat equation by the Lie symmetry sense. Since the fundamental solution and behavior analyses of heat equation can be found from the literature, representation of the Ho-Lee stochastic interest rate model into the heat equation makes it more easily to understand and interpret this stochastic differential equation. This is one of the main motivation of the this work. Although there has been important works about bond-pricing with respect to the Lie symmetry analysis of certain SDEs in financial mathematics, for instance Lie symmetry analysis of the Merton-Black Sholes, CIR and Vasicek models among others, to the best of our knowledge it has not been done for the Ho-Lee model yet. For this purpose, we try to do our best in this work to for this gap.

The remainder of the paper is organized as follows. In Section 2, we briefly introduce the Ho-Lee model and related PDE. In section 3, we represent some theorems for the linear $(1+1)$ parabolic PDEs using the invariant criteria. In Section 4, we show transformations from Ho-Lee model to the classical heat equation and find the fundamental solution of corresponding heat equation for Ho-Lee model under the invariant criteria. In Section 5, we illustrate importance of parameter selection for power functions in the drift term and analyze it with respect to financial usefulness. Section 6 concludes the paper.

\section{Bond pricing with Ho-Lee model}

Thomas S. Y. Ho and Sang-Bin Lee developed Ho-Lee model in 1986 (see [5]). It is a short rate model which is known as the first arbitrage free model of interest rates. It has also discrete-time and continuous-time versions. It is a useful model and has wide application area in mathematical finance such that bond pricing, option pricing and modeling future interest rate are just some of them in the literature.

The short interest rate under the continuous-time version follows a normal process and its stochastic differential equation (SDE) is as follow.

$$
d r(t)=b(t) d t+\sigma d W(t)
$$

Here, the non-stochastic drift term $b(t)$ is a function of time and it provides more flexibility in matching the model to real data. Its calibration to market price is the most important part at the applications so that it is usually chosen by observing market data. The diffusion term $\sigma$ is a constant while $r(t)$ and $W(t)$ show the interest rate and one-dimensional standard Brownian motion, respectively. On the other hand, the drift term has important role and should be determined carefully otherwise the interest rate can be reached to the negative values which is not expected at the financial applications.

Furthermore, Ho and Lee derived this model (see [5]) under the complete market assumptions (i.e. the market is frictionless, no taxes, no transaction costs, no arbitrage opportunity and all securities are perfectly divisible etc). Moreover, under these assumptions, $\mathrm{u}(\mathrm{x}, \mathrm{t})$, which is the zero coupon price in the Ho-Lee model, satisfies the scalar linear one time variable and one space variable parabolic PDE

$$
\frac{\partial u}{\partial t}+\frac{1}{2} \sigma^{2} \frac{\partial^{2} u}{\partial^{2} x}+b(t) \frac{\partial u}{\partial x}-x u=0, u(x, T)=1 .
$$

where $T$ represents the terminal time.

\section{Linear $(1+1)$ parabolic PDEs under the invariant criteria}

At this section, we briefly introduce the main results of Mahomed on the invariant characterization of scalar linear $(1+1)$ parabolic PDEs (for more details see [8]). 
The general representation of the scalar linear parabolic PDE of one time and one space variable is as follows.

$$
\frac{\partial u}{\partial t}=a(t, x) \frac{\partial^{2} u}{\partial x^{2}}+b(t, x) \frac{\partial u}{\partial x}+c(t, x) u,
$$

where the coefficients $a, b$ and $c$ are the continuous functions of $t$ and $x$.

Lie proved at his seminal works [6] in 1881 that a scalar linear parabolic PDE (3) has the four canonical forms.

$$
\begin{aligned}
& \frac{\partial u}{\partial t}=\frac{\partial^{2} u}{\partial x^{2}}, \\
& \frac{\partial u}{\partial t}=\frac{\partial^{2} u}{\partial x^{2}}+\frac{A}{x^{2}} u, \quad A \neq 0, \\
& \frac{\partial u}{\partial t}=\frac{\partial^{2} u}{\partial x^{2}}+c(x) u, \quad c \neq 0, A / x^{2}, \\
& \frac{\partial u}{\partial t}=\frac{\partial^{2} u}{\partial x^{2}}+c(t, x) u, \quad c \neq 0, A / x^{2} .
\end{aligned}
$$

The invariant criteria are provided with the following theorems (see [8]) which help to reduce the scalar linear $(1+1)$ parabolic PDEs (3) into 1'st Lie canonical form in Equation (4).

Theorem 1. [Mahomed, [8]]. The linear parabolic equation (3) is reducible to the classical heat PDE (or the first Lie canonical form) $\frac{\partial \bar{u}}{\partial \bar{t}}=\frac{\partial^{2} \bar{u}}{\partial \bar{x}^{2}}$ via the transformations

$$
\begin{aligned}
& \bar{t}=\phi(t), \\
& \bar{x}= \pm \int\left[\dot{\phi} a(t, x)^{-1}\right]^{\frac{1}{2}} d x+\beta(t), \\
& \bar{u}=v(t)[a(t, x)]^{-\frac{1}{4}} u \exp \left[\int \frac{b(t, x)}{2 a(t, x)} d x-\frac{1}{8} \frac{\ddot{\phi}}{\dot{\phi}}\left(\int \frac{d x}{a(t, x)^{\frac{1}{2}}}\right)^{2}-\frac{1}{2} \int \frac{1}{a(t, x)^{\frac{1}{2}}} \frac{\partial}{\partial t}\left(\int \frac{d x}{a(t, x)^{\frac{1}{2}}}\right) d x \pm \frac{1}{2} \frac{\dot{\beta}}{\dot{\phi}^{\frac{1}{2}}} \int \frac{d x}{a(t, x)^{\frac{1}{2}}}\right] .
\end{aligned}
$$

where $\dot{\phi}$ and $a$ have the same sign, and $\phi, \beta$ and $v$ satisfy

$$
\dot{\phi} \bar{c}=J+\frac{\partial}{\partial t} \int \frac{b(t, x)}{2 a(t, x)} d x-\frac{1}{2} \int \frac{1}{a(t, x)^{\frac{1}{2}}} \frac{\partial^{2}}{\partial t^{2}}\left(\int \frac{d x}{a(t, x)^{\frac{1}{2}}}\right) d x+f(t)\left(\int \frac{d x}{a(t, x)^{\frac{1}{2}}}\right)^{2}+g(t)\left(\int \frac{d x}{a(t, x)^{\frac{1}{2}}}\right)+h(t),
$$

with $J$ is

$$
J=c-\frac{b_{x}}{2}+\frac{b a_{x}}{2 a}+\frac{a_{x x}}{4}-\frac{3}{16} \frac{a_{x}^{2}}{a}-\frac{a_{t}}{2 a}-\frac{b^{2}}{4 a}
$$

and

$$
\begin{aligned}
& f(t)=\frac{1}{16} \frac{\ddot{\phi}^{2}}{\dot{\phi}^{2}}-\frac{1}{8}\left(\frac{\ddot{\phi}}{\dot{\phi}}\right)_{t}, \\
& g(t)= \pm \frac{1}{4} \frac{\ddot{\phi}}{\dot{\phi}} \frac{\dot{\beta}}{\dot{\phi}^{\frac{1}{2}}} \pm \frac{1}{2}\left(\frac{\dot{\beta}}{\dot{\phi}^{\frac{1}{2}}}\right)_{t}, \\
& h(t)=\frac{1}{4} \frac{\ddot{\phi}}{\dot{\phi}}+\frac{1}{4} \frac{\dot{\beta}^{2}}{\dot{\phi}}+\frac{\dot{v}}{v} .
\end{aligned}
$$

The functions $f, g$ and $h$ are constrained by the relation defined in equation (6) (see [8] and references therein).

Theorem 2. [Mahomed, [8]]. The statements in (a) and (b) are equivalent. 
(a) the coefficients of parabolic equation (3) satisfies the invariant equation

$$
2 L_{x}+2 M_{x}-N_{x}=0,
$$

where

$$
L=|a|^{\frac{1}{2}}\left[|a|^{\frac{1}{2}} J_{x}\right]_{x}, M=|a|^{\frac{1}{2}}\left[|a|^{\frac{1}{2}} \partial_{t}(b / 2 a)\right]_{x} \cdot N=|a|^{\frac{1}{2}} \partial_{t}^{2}\left(1 /|a|^{\frac{1}{2}}\right),
$$

and $J$ is given by equation (7);

(b) the linear parabolic equation (3) can be reduced to the heat PDE $\frac{\partial \bar{u}}{\partial \bar{t}}=\frac{\partial^{2} \bar{u}}{\partial \bar{x}^{2}}$ via the transformations (5) for which $\phi$, $\beta$ and $v$ are constructed from equations (8) with the functions $f, g$ and $h$ are constrained by the relation

$$
J+\frac{\partial}{\partial t} \int \frac{b(t, x)}{2 a(t, x)} d x-\frac{1}{2} \int \frac{1}{a(t, x)^{\frac{1}{2}}} \frac{\partial^{2}}{\partial t^{2}}\left(\int \frac{d x}{a(t, x)^{\frac{1}{2}}}\right) d x+f(t)\left(\int \frac{d x}{a(t, x)^{\frac{1}{2}}}\right)^{2}+g(t)\left(\int \frac{d x}{a(t, x)^{\frac{1}{2}}}\right)+h(t)=0 .
$$

\section{Fundamental solution of Ho-Lee parabolic PDE}

In this section, we find the transformation of Ho-Lee PDE in equation (2) for the related Lie canonical forms by using the given theorems in section 3. Then, we obtain fundamental solution for the corresponding Lie canonical form, which is the classical heat equation, with respect to the invariant criteria approach.

\subsection{Transformation from Ho-Lee model to the heat equation}

The related coefficients can be written easily, when we compare the Ho-Lee PDE (2) with the scalar linear, one time and one space variable parabolic PDE (3), as follows:

$$
\begin{aligned}
& a(t, x)=-\frac{1}{2} \sigma^{2}, \\
& b(t, x)=-b(t), \\
& c(t, x)=x .
\end{aligned}
$$

The coefficient $a(t, x)$ is a constant and the $b(t, x)$ is independent of $x$, so that we get $M=N=0$ (see equation (10)). Moreover, we evaluate $J$ for Ho-Lee PDE using the Theorem 2's condition which is given by equation (7):

$$
J=x+\frac{b^{2}(t)}{2 \sigma^{2}}
$$

Similarly, we obtain $L=0$ by using this $J$ in equation (10). If we substitute these values in the invariant condition (9) in Theorem 2, then it shows that invariant condition is satisfied. So that, we can reduce Ho-Lee PDE to the classical heat equation since all the statements of Theorem 2 are equivalent. For this purpose, we need to obtain the transformations, which is defined by Theorem 1, by using the coefficients given in equation (12). First of all, we get the following functions defined in Theorem 2:

$$
f(t)=0, g(t)=-\frac{\sigma}{\sqrt{2}}+\frac{b^{\prime}(t)}{\sqrt{2} \sigma}, h(t)=-\frac{b^{2}(t)}{2 \sigma^{2}}
$$


Then, we use these functions and obtain the following functions defined in Theorem 1 :

$$
\begin{aligned}
& \phi(t)=-\frac{c_{2}^{2}}{t}+c_{3} \\
& \beta(t)=\frac{c_{2}}{2}\left(\frac{\sqrt{2}}{\sigma}-1\right) \int \frac{b(t)}{t} d t+\frac{c_{2}}{2 t} \int b(t) d t-\frac{c_{2} \sigma}{2 \sqrt{2}} t+c_{6} \frac{1}{t}+c_{7} \\
& v(t)=\exp \left\{\int\left(\frac{1}{2 t}-\frac{1}{16 t^{2}}\left[\frac{\sqrt{2}}{\sigma}\left(t b(t)-\int b(t) d t\right)-\sqrt{2} \sigma\left(\frac{1}{2} t^{2}\right)+c_{5}\right]^{2}-\frac{b^{2}(t)}{2 \sigma^{2}}\right) d t\right\}
\end{aligned}
$$

where $c_{i}$ 's are integral constants. After that, we get the transformations which reduce the Ho-Lee PDE into the heat equation as below.

$$
\begin{aligned}
\bar{t} & =-\frac{c_{2}^{2}}{t}+c_{3} \\
\bar{x} & = \pm \frac{\sqrt{2} c_{2}}{\sigma t} x+\frac{c_{2}}{2}\left(\frac{\sqrt{2}}{\sigma}-1\right) \int \frac{b(t)}{t} d t+\frac{c_{2}}{2 t} \int b(t) d t-\frac{c_{2} \sigma}{2 \sqrt{2}} t+c_{6} \frac{1}{t}+c_{7} \\
\bar{u} & =\exp \left\{\int\left(\frac{1}{2 t}-\frac{1}{16 t^{2}}\left[\frac{\sqrt{2}}{\sigma}\left(t b(t)-\int b(t) d t\right)-\sqrt{2} \sigma\left(\frac{1}{2} t^{2}\right)+c_{5}\right]^{2}-\frac{b^{2}(t)}{2 \sigma^{2}}\right) d t\right\}\left[-\frac{1}{2} \sigma^{2}\right]^{-\frac{1}{4}} u \\
& \exp \left\{\frac{b(t)}{\sigma^{2}} x+c_{8}+\frac{1}{4 t}\left(\frac{\sqrt{2}}{\sigma} x+c_{9}\right)^{2} \pm \frac{1}{4 t}\left[\frac{\sqrt{2}}{\sigma}\left(t b(t)-\int b(t) d t\right)-\sqrt{2} \sigma\left(\frac{1}{2} t^{2}\right)+c_{5}\right]\left(\frac{\sqrt{2}}{\sigma} x+c_{9}\right)\right\}
\end{aligned}
$$

where $c_{i}$ 's are integral constants. Existing of the transformations represent with the equations in (16) means that we can reduce Ho-Lee PDE into the classical heat equation. Now, we are at the position to use the advantage of the heat equation since the fundamental solution of the classical heat equation is well-known in the literature. For instance, Pooe, Mahomed and Soh gave its solution in "barred" coordinates in their paper (see [12]) as follows.

$$
\bar{u}=\frac{1}{2 \sqrt{\bar{t} \pi}} \exp \left[-\frac{\bar{x}^{2}}{4 \bar{t}}\right] .
$$

If we substitute the equation (18) in the equation (16) and solve it for $u$ then we have the fundamental solution of Ho-Lee PDE (2):

$$
\begin{aligned}
u(x, t) & =\frac{1}{2 \sqrt{\bar{t} \pi}} \exp \left[-\frac{\bar{x}^{2}}{4 \bar{t}}\right] \exp ^{-1}\left\{\int \left(\frac{1}{2 t}-\frac{1}{16 t^{2}}\left[\frac{\sqrt{2}}{\sigma}\left(t b(t)-\int b(t) d t\right)-\sqrt{2} \sigma\left(\frac{1}{2} t^{2}\right)+c_{5}\right]^{2}\right.\right. \\
& \left.\left.-\frac{b^{2}(t)}{2 \sigma^{2}}\right) d t\right\}\left[-\frac{1}{2} \sigma^{2}\right]^{\frac{1}{4}} \exp ^{-1}\left\{\frac{b(t)}{\sigma^{2}} x+c_{8}+\frac{1}{4 t}\left(\frac{\sqrt{2}}{\sigma} x+c_{9}\right)^{2} \pm \frac{1}{4 t}\left[\frac{\sqrt{2}}{\sigma}\left(t b(t)-\int b(t) d t\right)\right.\right. \\
& \left.\left.-\sqrt{2} \sigma\left(\frac{1}{2} t^{2}\right)+c_{5}\right]\left(\frac{\sqrt{2}}{\sigma} x+c_{9}\right)\right\}
\end{aligned}
$$

Although the left hand side of the solution does not depend on barred parameters, the $\bar{x}$ and $\bar{t}$ still appears at the right hand side of it (19). Therefore, we need to substitute the $\bar{x}$ and $\bar{t}$ values from the equation (16) into of it, then the fundamental solution of Ho-Lee PDE becomes 


$$
\begin{aligned}
u(x, t) & =\frac{1}{2 \sqrt{\left(-\frac{c_{2}^{2}}{t}+c_{3}\right) \pi}} \exp \left[-\frac{\left( \pm \frac{\sqrt{2} c_{2}}{\sigma t} x+\frac{c_{2}}{2}\left(\frac{\sqrt{2}}{\sigma}-1\right) \int \frac{b(t)}{t} d t+\frac{c_{2}}{2 t} \int b(t) d t-\frac{c_{2} \sigma}{2 \sqrt{2}} t+c_{6} \frac{1}{t}+c_{7}\right)^{2}}{4\left(-\frac{c_{2}^{2}}{t}+c_{3}\right)}\right] \\
& \exp ^{-1}\left\{\int\left(\frac{1}{2 t}-\frac{1}{16 t^{2}}\left[\frac{\sqrt{2}}{\sigma}\left(t b(t)-\int b(t) d t\right)-\sqrt{2} \sigma\left(\frac{1}{2} t^{2}\right)+c_{5}\right]^{2}-\frac{b^{2}(t)}{2 \sigma^{2}}\right) d t\right\}\left[-\frac{1}{2} \sigma^{2}\right]^{\frac{1}{4}} \\
& \exp ^{-1}\left\{\frac{b(t)}{\sigma^{2}} x+c_{8}+\frac{1}{4 t}\left(\frac{\sqrt{2}}{\sigma} x+c_{9}\right)^{2} \pm \frac{1}{4 t}\left[\frac{\sqrt{2}}{\sigma}\left(t b(t)-\int b(t) d t\right)-\sqrt{2} \sigma\left(\frac{1}{2} t^{2}\right)+c_{5}\right]\right. \\
& \left.\left(\frac{\sqrt{2}}{\sigma} x+c_{9}\right)\right\}
\end{aligned}
$$

\section{Illustration for the drift term of Ho-Lee model: $b(t)=k t^{\alpha}$}

As it is seen in the equation (20), the fundamental solution of Ho-Lee PDE differs with the drift term $b(t)$ of Ho-Lee model. We deal with the importance of its calibration in section 2 and take your attention on choosing of it so that we can avoid from negative interest rate values which are not expected at the financial applications.

At this section, for an example, we choose the drift term from the power functions as $b(t)=k t^{\alpha}$ where $k$ and $\alpha$ are constants. Then, we illustrate several situations and present some results for the related parameters of the selected drift term by using the numerical solution of stochastic differential equations [11] via Milstein method [10] at the simulations. We try to make its parameters' selection more easily under the light of the simulation results. On the other hand, the fundamental solution of corresponding heat equation can be obtained for Ho-Lee model with this drift term by using the equation in (20).

For instance, we choose the following parameters when we perform simulations for the Ho-Lee model.

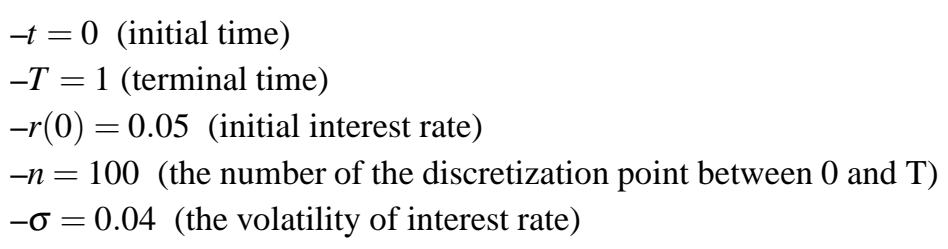

If $k$ takes positive values $(k>0)$, then the interest rate generally increases. Otherwise, it decreases and can be reached to the negative values which are meaningless by the financial aspect. The latter case is illustrated in the both panels of figure 1. Therefore, practitioners should choose positive values of $k$ when they use Ho-Lee interest rate model at their analyses. Although the interest rate increases relatively for all $\alpha \in \mathbb{R}$ and $k>0$, we can control its increasing by choosing suitable $\alpha$ parameter. As an example, it increases rapidly and may be overvalued when $\alpha=0.5$ (see left panel of the figure 2) which is not expected by the investor at the stable market (i.e. except crisis or any other abnormal situations). For this reason, we need to choose more useful value of $\alpha$ (i.e. $\alpha=-0.5$, see right panel of figure 2). This is nothing more than fundamental calculus result since the properties of the $t^{\alpha}$ functions are well-known. All the analysis results show that interest rate relatively diverge from the initial value at the terminal time. Although small or relatively big magnitude changes in interest rate can be reasonable, simulation results show that large fluctuations happen during the time. Especially, it reaches to 1.5 at the terminal time which is the approximately 300 times of the initial value 0.05 (see left panel of figure 2). Since the Ho-Lee model does not have mean reversion property as Cox, Ingersol and Ross (CIR) model [1] has, its application results may be misled to the market players. But the more optimistic scenario can be obtained with positive $k$ and negative $\alpha$ pairs if we want to control its changes around the initial interest rate. Since the estimation of interest rate behavior may help for your analysis to be more realistic by the time. Here, we present some optimistic scenario results in figure 3. 

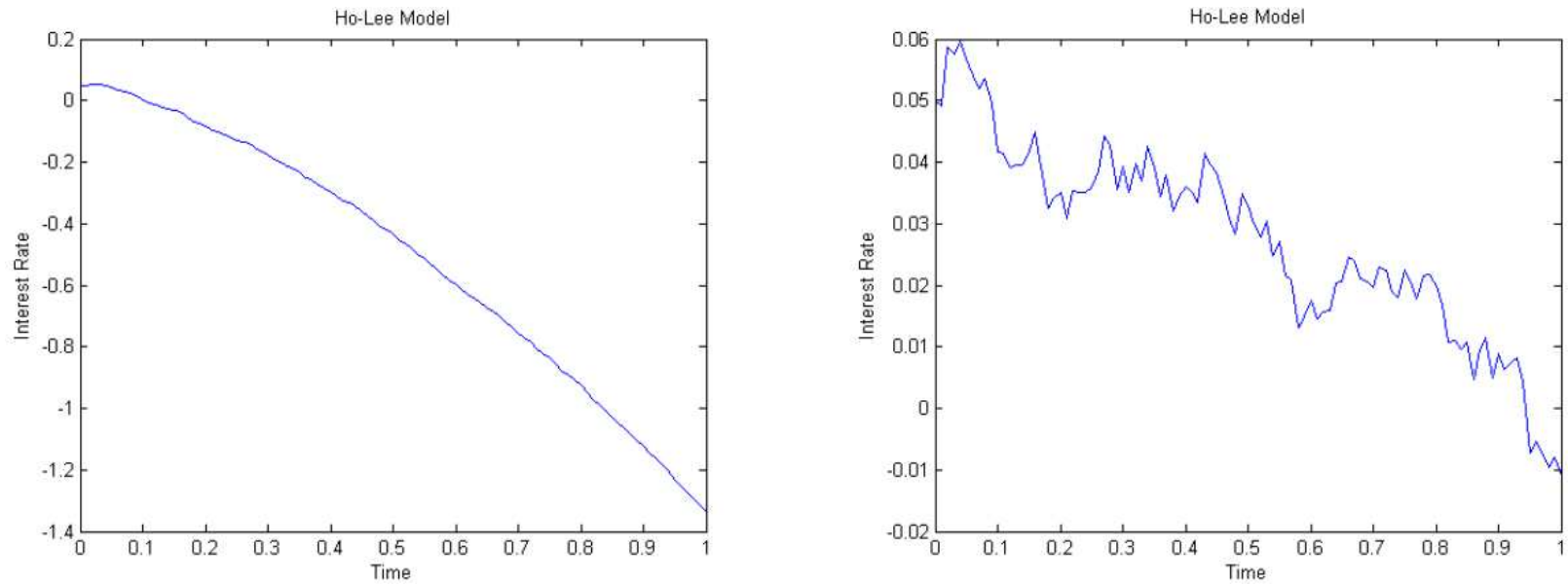

Fig. 1: Behavior of interest rates for $k=-0.2$ when $\alpha=0.5$ (left) and $\alpha=-0.5$ (right).
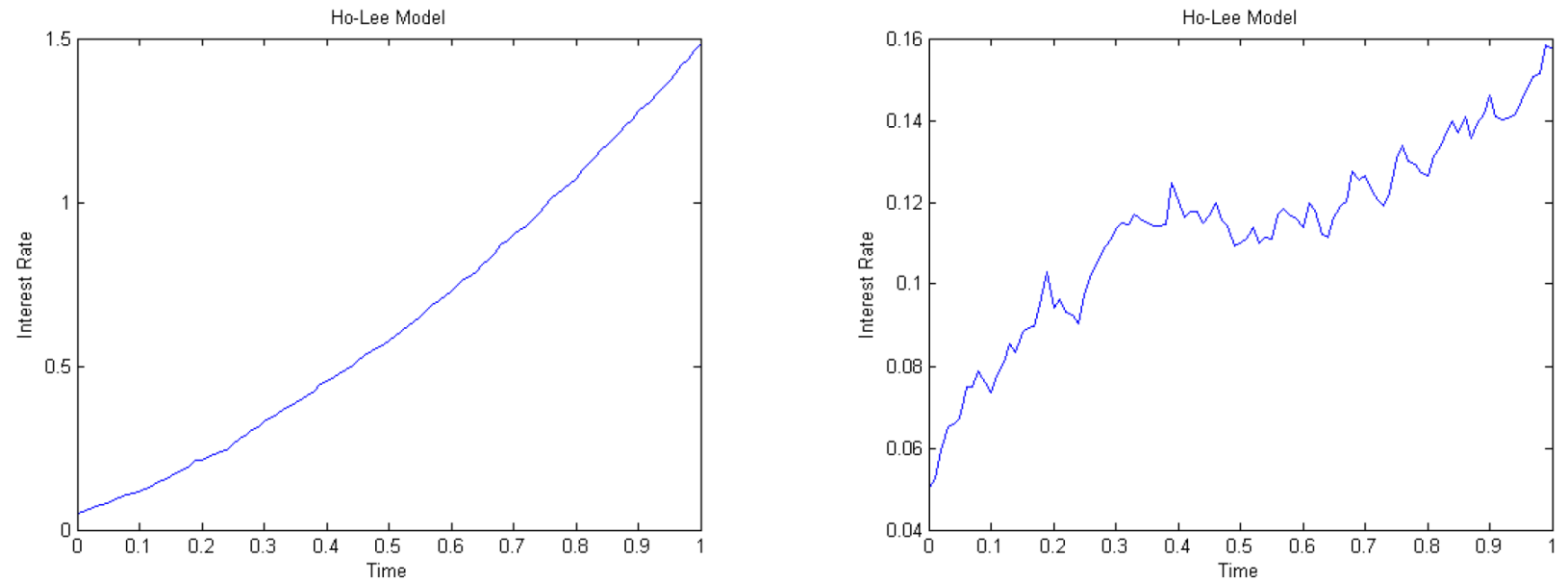

Fig. 2: Behavior of interest rates for $k=0.2$ when $\alpha=0.5$ (left) and $\alpha=-0.5$ (right).
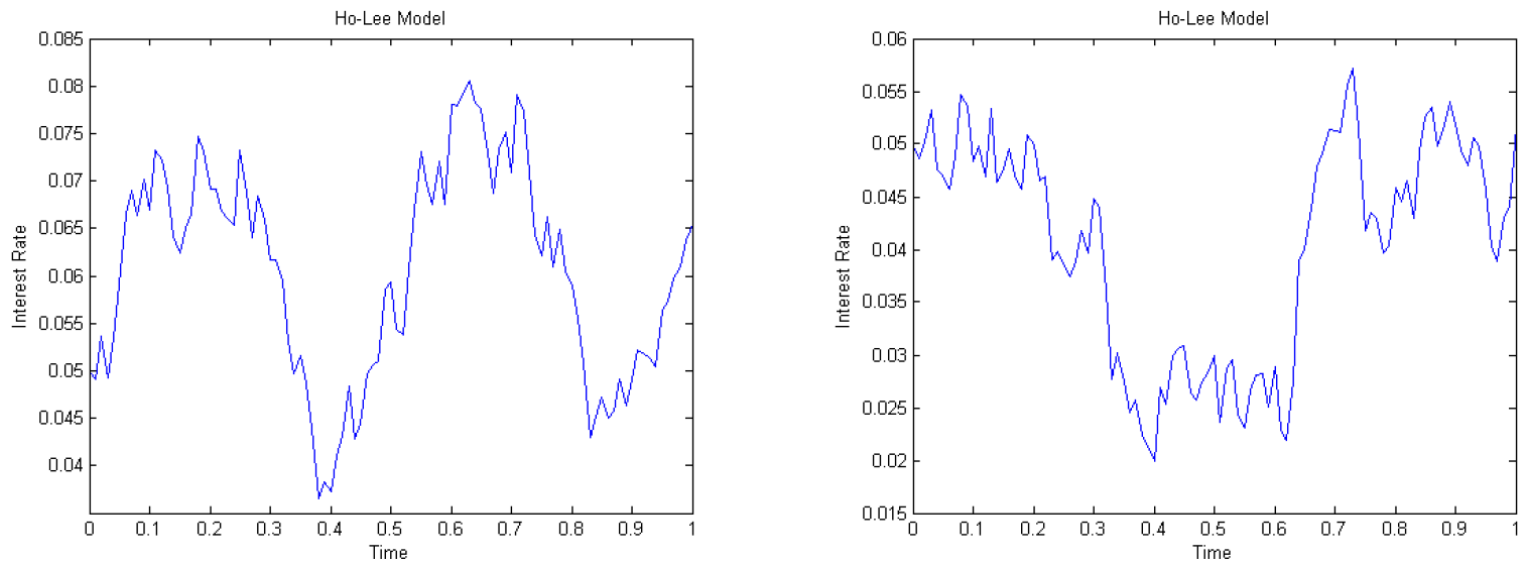

Fig. 3: Behavior of interest rates for $k=0.2$ when $\alpha=-0.7$ (left) and $\alpha=-0.8$ (right). 


\section{Conclusions}

The numerical solution of stochastic differential equations may take long time which depends on either complexity of the SDEs being considered or the numerical solution method is used. Therefore, it is challenging to find the fundamental solution of SDEs by using the invariant criteria in order to convert underlying SDE to the one of the Lie canonical forms. We present the transformations from linear $(1+1)$ parabolic PDE for the Ho-Lee model to the 1'st Lie canonical form which is also identical to the classical heat equation. Thus, we obtain the fundamental solution of the corresponding PDE for the Ho-Lee model based on this transformations by using the advantage of the heat equation.

Moreover, the drift term of the Ho-Lee model is a deterministic function of time and it provides more flexibility in matching the model to real data. Its calibration to market price is the most important part at the applications so that it is usually chosen by observing market data. For this purpose, we perform extensive simulations and obtain important results for the calibration of it. Such that, we present drift term's calibration for power function with respect to its parameter analysis.

After that, we can use the analytical solution for the corresponding heat equation instead of the numerical solution of SDE's at the financial applications. At this step, we notice the existence of a problem "which approximation to the solution of SDE is more efficient than others?" by the time perspective since the investment timing takes very important role for the practitioners at the financial markets. We do not consider to find the answer of this question in this paper and its answer is not considered by the other authors who used the invariant criteria approximations for the certain SDEs in mathematical finance. We believe that the answer of this problem in terms of their trade-offs, which is still an open problem, should be a subject of the different research paper.

\section{Authors' contributions}

All authors have contributed to all parts of the article. All authors read and approved the final manuscript.

\section{References}

[1] J.C. Cox, J.E. Ingersoll and S.A. Ross, A Theory of the Term Structure of Interest Rates, Econometrica, 53, 385-407, 1985.

[2] S. Dimas, K. Andriopoulos, D. Tsoubelis and P.G.1. Leach, Complete Specification of Some Partial Differential Equations That Arise in Financial Mathematics, J. Nonlinear Math. Phys., 73-92, 2009.

[3] R.K. Gazizov and N.H. Ibragimov, Lie Symmetry Analysis of Differential Equations in Finance, Nonlinear Dynam. 17(4), 387-407, 1998.

[4] J. Goard, New solutions to the bond-pricing equation via Lie's Classical Method, Math. Comput. Model., 32, 299-313, 2000.

[5] T. S. Y. Ho and S.-B. Lee, Term Structure Movements and Pricing of Interest Rate Claims, Journal of Finance, 41, 1011-1029, 1986.

[6] S. Lie, On integration of a Class of linear partial differential equations by means of definite integrals Archiv for Mathematik $1 \mathrm{~g}$ Naturvidenskab, VI(3) 328-368, 1881 [in German]. Reprinted in S.Lie, Gesammelte Abhadlundgen, 3 papers, XXXV.

[7] F.M. Mahomed, K.S. Mahomed, R. Naz and E. Momoniat, Invariant Approaches to Equations of Finance, Math. Comput. Appl., 18(3), 244-250, 2013.

[8] F.M. Mahomed, Complete Invariant Characterization of Scalar Linear (1+1) Parabolic Equations, J. Nonlinear Math. Phys., 15, 112-123, 2008.

[9] R. Merton, Option Pricing when Underlying Stock Returns are Discontinuous, J. Financial Economics, 3, 125-144, 1976.

[10] G.N. Milstein, Approximate Integration of Stochastic Differential Equations, Theor. Prob. Appl. 19: 557-562, 1974.

[11] P.E. Kloeden, E. Platen, and H. Schurz, Numerical Solution of SDE Through Computer Experiments, Springer, Berlin, 2003.

[12] C.A. Pooe, F.M. Mahomed and C. Wafo Soh, Fundamental solutions for zero-coupon bond pricing models, Nonlinear Dynam., 36, 69-76, 2004.

[13] W. Sinkala, P.G.L. Leach and J. G. O'Hara, Zero-coupon Bond Prices in Vasicek and CIR Models, Math. Meth. Appl. Sci., 31, 665-678, 2008. 